\title{
Electrokinetic treatments of soils: potential for geoenergy applications
}

\author{
Andony Landivar Macias ${ }^{1}$ and Alessandro F. Rotta Loria ${ }^{1, *}$ \\ ${ }^{1}$ Northwestern University, Department of Civil and Environmental Engineering, Mechanics and Energy Laboratory \\ 2145 Sheridan Road, Evanston, Illinois 60208, United States of America
}

\begin{abstract}
The application of a direct current electric field to porous materials such as soils in the form of an electrokinetic treatment induces a variety of multiphysical phenomena. Critical phenomena involved during an electrokinetic treatment of soils are electrical migration (i.e., movement of ions), electroosmosis (i.e., movement of water), electrolysis (i.e., breakdown of chemical compounds), and electrochemical reactions (i.e., oxidation/reduction and precipitation/mineralization reactions). Such phenomena lead to changes in the structure of soils. As changes in the structure of soils induce variations in their properties and behaviors (e.g., thermo-hydro-mechanical), electrokinetic treatments represent resourceful means to address broad geoengineering challenges. The purpose of this paper is to highlight the potential of electrokinetic treatments as versatile techniques capable of providing solutions for diverse geoengineering applications via a controllable change of the properties and behaviors of soils. From this perspective, this work specifically encompasses electrokinetically induced phenomena in soils and expands on opportunities in the context of geoenergy applications, among others, which can be mediated by electrokinetic treatments.
\end{abstract}

\section{Introduction}

Porous materials such as soils are ubiquitous in the context of infrastructures, natural hazards and energy resources. For instance, soils play an essential role as foundation materials for structures, critically controlling their durability and safety. Soils often represent the causative of natural hazards, governing the magnitude of such events. Yet, soils regulate the effectiveness of geoenergy storage and extraction applications. Given the central role of soils in the previous contexts, the ability to control the properties and behaviors of such materials (e.g., thermo-hydro-mechanical) seems critical.

Soils are complex multiphase materials typically constituted by solid particles, one or more pore fluids (e.g., water and air) interacting together, and ions [1]. The wide composition and formation environments of soils lead to different properties and behaviors of such materials through their structure. In many situations, inadequate soil properties and behaviors are encountered at sites, consequently requiring treatments or manipulations to achieve engineering goals [2]. Developing effective and sustainable techniques to modify the properties and behaviors of soils is paramount for addressing geoengineering challenges and meeting human activity needs.

A principal purpose of in situ ground improvement techniques is to modify (e.g., enhance) the engineering qualities of soils, intended as the properties and behaviors of such materials, for ensuring the performance of a variety of applications. Examples of interests include the enhancement of the capabilities of soils to sustain mechanical loads, to prevent fluids permeation, or to transfer heat. Examples of ground improvement techniques include conventional treatments such as drainage $[3,4]$ or compaction and preloading $[3,4]$, as well as more novel treatments such as grouting [2] and bioremediation [5]. However, all of these treatments can have drawbacks. Conventional treatments may be inapplicable in situations of limited accessibility. More novel treatments can affect the chemical and/or biological state of the soil or groundwater.

Electrokinetic treatments, which involve the application of a low-voltage, direct current (DC) electric field to soils and other porous materials, represent effective, environmentally friendly and versatile solutions for addressing engineering challenges that involve or require ground improvement. The reason for this is that electrokinetic treatments cause multiphysical phenomena (e.g., thermo-hydro-mechanical) in soils, which by inducing variations in the structure of such materials also influence their properties and behaviors. This concept paper aims at elucidating the multiphysical phenomena caused by electrokinetic treatments in soils, and a number of influences on soil behavior.

In the following, the fundamental concepts and theoretical background of electrokinetically induced phenomena in soils are presented first. Next, a focus is given to microscopic changes in structure of soils induced by such phenomena and the related effects on their properties. Then, a discussion of engineering applications that can benefit electrokinetic treatments are

\footnotetext{
* Corresponding author: af-rottaloria@northwestern.edu
} 
reported, and comments on opportunities for geoenergy applications are reported. Finally, concluding remarks that can be drawn from this work are summarized.

\section{Fundamentals of electrokinetic phenomena in soils}

\subsection{General}

Under a low-voltage DC electric field between two electrodes (i.e., anode and cathode), soils undergo physical and chemical changes due to electrokinetically induced phenomena [4]. Critical phenomena involved with an electrokinetic treatment of soils are electrical migration (i.e., movement of ions), electroosmosis (i.e., movement of water), electrolysis (i.e., breakdown of chemical compounds), electrochemical reactions (i.e., oxidation/reduction and precipitation/mineralization reactions), and Joule heating. These phenomena act in tandem, modifying the soil structure and affecting the properties and behaviors of soils, similar to other relevant porous materials that may be subjected to treatment.

\subsection{Electrical migration and electroosmosis}

Under a DC electric field, free ions in an electrolyte solution (e.g., water with ions) are subjected to Coulomb electrostatic forces, migrating toward the electrodes and leading to the phenomenon of electrical migration [6]. Positive ions (i.e., cations) move toward the negative cathode. Negative ions (i.e., anions) move toward the positive anode. In soils, ions are usually present in the pore fluid. While moving under an electric field, ions can carry with them water molecules across the pore network. The dual migration of cations and water molecules due to the influence of an electric field is called electroosmosis [7]. A schematic of the considered phenomena is presented in Figure 1.

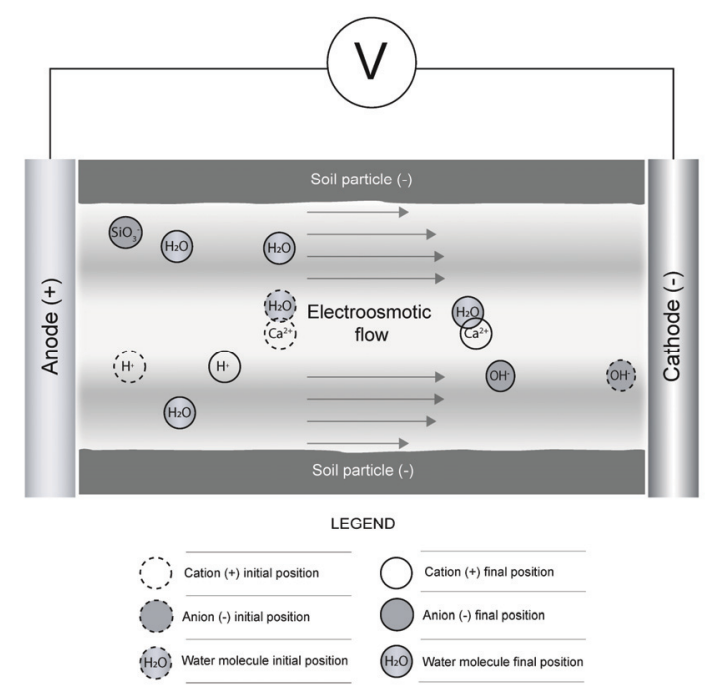

Fig. 1. Electroosmosis and electrical migration in soils.
Over the past sixty years, electroosmosis has been the most studied phenomenon associated with electrokinetic treatments due to its capability to remediate and consolidate natural slopes and general soil masses [8,9]. Through electroosmotic consolidation, soils experience a mass transfer of pore water, leading to desaturation, which can be otherwise achieved through mechanical consolidation. Consequently, soil particles are packed closer together, becoming apparently overconsolidated, and the material is likely to be characterized by changes in strength and stiffness, thermal conductivity, and hydraulic conductivity [4].

\subsection{Electrolysis}

Under a DC electric field, electrochemical changes are commenced with chemical reactions. Voltage triggers a non-spontaneous chemical decomposition called electrolysis as follows [10]

Anode: $2 \mathrm{H}_{2} \mathrm{O}(\mathrm{l})-4 \mathrm{e}^{-} \rightarrow \mathrm{O}_{2}(\mathrm{~g})+4 \mathrm{H}^{+}(\mathrm{aq})$
Cathode: $4 \mathrm{H}_{2} \mathrm{O}(\mathrm{l})+4 \mathrm{e}^{-} \rightarrow 2 \mathrm{H}_{2}(\mathrm{~g})+4 \mathrm{OH}^{-}(\mathrm{aq})$

The decomposition of water into $\mathrm{H}^{+}$and $\mathrm{OH}^{-}$ions leads to the formation of acid and alkaline fronts near the anode and cathode, respectively. Due to the applied electric field, the acid and alkaline fronts will migrate across the pore network, dissolving and desorbing other ionic species in the soil [11]. These fronts move until meeting once again for the following neutralization reaction that slowly recovers the initial $\mathrm{pH}$ of the soil.

$\mathrm{H}^{+}(\mathrm{aq})+\mathrm{OH}^{-}(\mathrm{aq}) \leftrightarrow \mathrm{H}_{2} \mathrm{O}(\mathrm{l})$

\subsection{Oxidation and reduction reactions}

Under a DC electric field, products of chemical decompositions can be introduced in the soil, thus altering the composition and mineralogy of the material $[12,13]$. Electrodes, for example, can degrade over time due to oxidation and reduction, affecting the efficiency of the treatment and introducing new ionic species into the pore fluid according to the following

$$
\begin{aligned}
& \text { Anode oxidation: } \mathrm{Me} \rightarrow \mathrm{Me}^{\mathrm{n}+}+\mathrm{ne}^{-} \\
& \text {Cathode reduction: } \mathrm{Me}^{\mathrm{n}+}+\mathrm{ne}^{-} \rightarrow \mathrm{Me} \downarrow
\end{aligned}
$$

The choice of electrode materials is thus crucial for the effectiveness of electrokinetic treatments. The key properties of interest for the electrode material are electrical conductivity, chemical reactivity, heat resistance and cost. High electrical conductivity allows applying the electric field to soils without losing voltage and keeping low energy consumption [14]. Low chemical reactivity allows applying the electric field to soils without altering the chemical composition of the material $[11,13]$. High heat resistance allows applying the electric field to soils without overheating them. Low cost of the electrodes allows for economical electrokinetic treatments [15]. 


\subsection{Mineralization reactions}

A DC electric field also facilitates chemical reactions between soil particles, pore fluid and free ions (i.e., ions not strongly attached to any soil particle). Such chemical reactions result in the mineralization of compounds that act as binding agents for particles and effectively change the structure of the soil $[12,13]$. This process is called electrokinetically induced mineral precipitation, equivalently termed mineralization. Figure 2 shows a schematic of electrokinetically mineralized crystals in a soil. Mineralization reactions can be effectively triggered in fine-grained soils due to the multitude of ions inherently present in such materials. These reactions, however, can also be triggered in coarse-grained soils in the presence of ions, which may naturally or artificially characterize such materials (through the use injection of engineered fluids during the electrokinetic treatment) [16-19].

Mineralization reactions have been highlighted in conjunction with electroosmosis. Yet, dewatering can be prevented by supplying water during the electrokinetic treatment to achieve mineralization reactions only [20]. Engineered fluids can be used to achieve mineralization reactions more effectively. Possible solutions that can be used for such purpose comprise, for instance, of calcium chloride, $\mathrm{CaCl}_{2}$, sodium silicate, $\mathrm{Na}_{2} \mathrm{SiO}_{3}$, and ammonium carbonate, $\left(\mathrm{NH}_{4}\right)_{2} \mathrm{CO}_{3} \quad[16,19]$. The aforementioned compounds dissociate in the pore fluid, breaking down to their ionic components by dissolution or electrolysis [10]. The resulting ions, just like the hydrogen and hydroxide ions, migrate under the influence of the applied electric field due to Coulomb electrostatic forces. As these ionic species move, they can react with other ionic species in the solid matrix of soil and the pore fluid, leading to mineralization reactions. Consequently, crystals such as calcium silicate hydrate, $\mathrm{CSH}\left(\mathrm{CaSiO}_{3} \cdot \mathrm{H}_{2} \mathrm{O}\right)$, or calcium-based compounds such as calcium carbonate, $\mathrm{CaCO}_{3}$, can be formed. Mineral crystal products consequently fill the material pores, altering the related structure. For example, the altered pore network due to mineralized crystals is subjected to a variation in porosity and surface contacts between the particles. Among other influences, this structural change can cause changes in density, stiffness, strength, hydraulic conductivity and thermal conductivity of the treated material $[4,21]$.

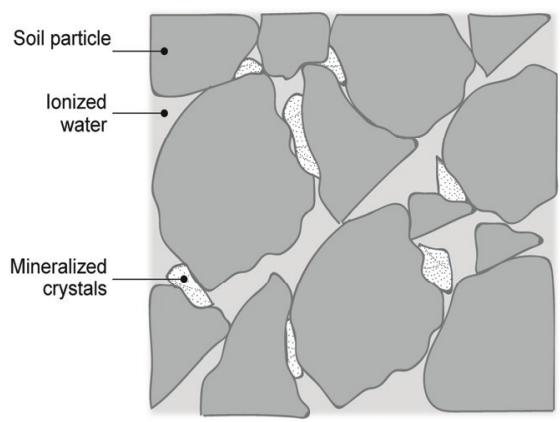

Fig. 2. Electrokinetically induced mineralization in a coarsegrained soil

\subsection{Joule heating}

A DC electric field applied to geomaterials results in a migration of electrons between the electrodes. The consequential heating caused by the collision of electrons with material particles, which is proportional to the electrical resistivity of the material, results in the phenomenon called Joule heating. Joule heating is the main thermal phenomenon involved in electrokinetic treatments, and is often considered as a side-effect (widely unexplored) of the process.

\section{Discussion and perspectives}

Electrokinetic treatments involve coupled multiphysical phenomena, which allow for a change in the structure of soils and other relevant porous materials. The achievable changes in the structure of soils through electrokinetic treatments are linked to changes in the properties and behaviors of such materials. A summary of these changes is presented in Figure 3.

\section{Electrokinetic treatment}

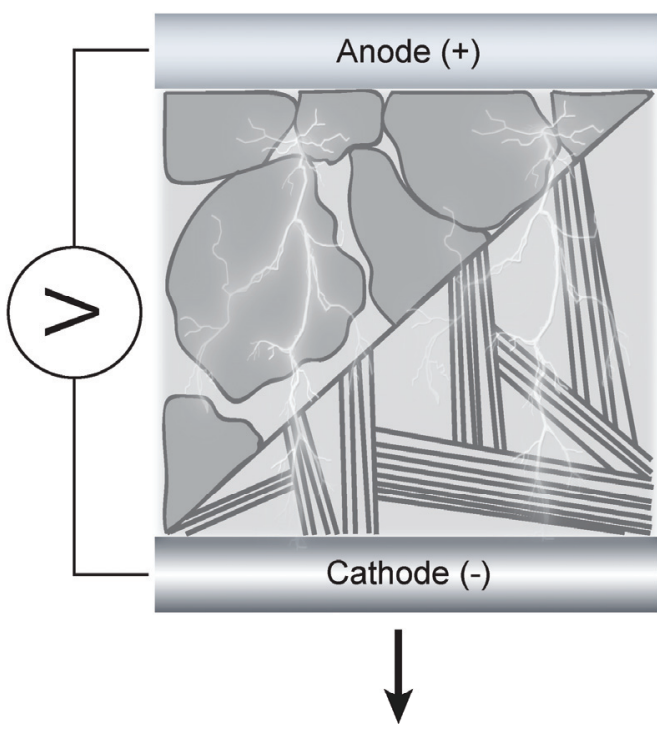

Multiphysical engineering effects

Variation in:
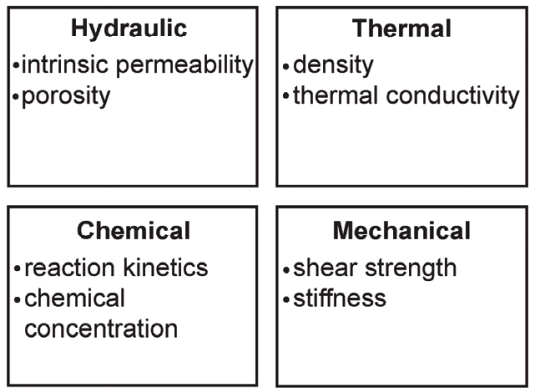

Mechanical - shear strength -stiffness

Fig. 3. Expected multiphysical effects triggered by electrokinetic treatments in soils 
The possibility to modify and tailor the engineering qualities of soils makes electrokinetic treatments unique candidates to serve diverse engineering applications across the fields of geomechanics, energy, and environmental sciences and engineering. Nevertheless, to date, relatively scarce investigations have analyzed the influence of DC electric fields on the properties and behaviors of such materials as compared to the significant and broad potential of electrokinetic treatments to enhance the performance of geoenergy applications, and shape, conserve and develop the natural and built environments. Premises in these contexts are reported below.

Electrokinetic treatments, through mineralization, can aid the efficiency of geoenergy harvesting applications. The increased contact between particles achieved through mineralization can increase the thermo-physical properties of soils, such as thermal conductivity and density. This influence allows for a more effective heat transfer across the treated materials, which can facilitate the extraction and injection of thermal energy through the ground. Promising potential relies in these applications for shallow and deep geothermal systems.

Electrokinetic treatments, through dewatering facilitated by electrical migration and electroosmosis, and/or mineralization, can change the hydraulic properties of soils, such as intrinsic permeability. Varying the intrinsic permeability of soils and other porous materials can be beneficial for flooding control. Dams, levees and water reservoirs are critical flood risk management structures that need constant conditioning and reassessment to be able to perform safely and adequately. Electrokinetic treatments may be employed to engineer and retrofit these structures by ensuring that they perform as expected.

Electrokinetic treatments, through dewatering or mineralization, can also influence the mechanical properties of targeted materials, such as shear strength and stiffness. Consolidation aided by electrosmosis leads to soil particles arranged in a denser configuration, leading to a tighter soil skeleton with an enhanced capability to sustain loads. Meanwhile, mineral crystals can also provide additional interparticle friction, which can further enhance the stiffness and shear strength of the material. A treated soil can become so hard after an electrokinetic treatment that it can resemble soft rock [22]. This outcome of electrokinetic treatments has the potential to be effectively employed for preventing natural hazards and protecting infrastructures.

Although the previous applications of electrokinetics seem promising, the understanding of several among the aforementioned phenomena (and the related effects on material properties and behavior) remains elusive. Theoretical and experimental research on this subject can significantly advance science and engineering, with rich potential for future discoveries and breakthroughs.

\section{Concluding remarks}

This work provided an overview of fundamental aspects involved with electrokinetically induced phenomena in soils. To date, electrokinetic phenomena in soils have been addressed in a number of studies, but still present various knowledge gaps. Resolution of these knowledge gaps can have significant implications for both science and engineering, with paramount applications for the enhancement of geoenergy applications, as well as for the conservation and development of the natural and built environments. Research is underway at the Mechanics and Energy Laboratory of Northwestern University to push a number of the current knowledge boundaries arising in this scope.

\section{References}

[1] K. Soga, S.A. Jefferis, Soil science and interdisciplinary aspects of geotechnical engineering, in: The Essence of Geotechnical Engineering: 60 Years of Géotechnique, Thomas Telford Publishing, 2008: pp. 133-140.

[2] J.K. Mitchell, In-Place Treatment of Foundation Soils, Journal of the Soil Mechanics and Foundations Division. 96 (1970) 73-110.

[3] K. Terzaghi, R.B. Peck, G. Mesri, Soil mechanics in engineering practice, Wiley, 1996.

[4] J.K. Mitchell, K. Soga, Fundamentals of soil behavior, Wiley, New York, 2005.

[5] D. Terzis, L. Laloui, A decade of progress and turning points in the understanding of bio-improved soils: A review, Geomechanics for Energy and the Environment. 19 (2019) 100116.

[6] L. Casagrande, Electro-osmosis in Soils, Géotechnique. 1 (1949) 159-177.

[7] D.H. Gray, J.K. Mitchell, Fundamental aspects of electro-osmosis in soils, Journal of the Soil Mechanics and Foundations Division. 93 (1967) 209-236.

[8] L. Casagrande, Electro-osmotic stabilization of soils, Journal of the Boston Society of Civil Engineers, ASCE. 39 (1952) 51-83.

[9] L. Bjerrum, J. Moum, O. Eide, Application of electro-osmosis to a foundation problem in a Norwegian quick clay, Geotechnique. 17 (1967) 214-235.

[10] T.L. Brown, H.E. LeMay, B.E. Bursten, C.J. Murphy, P.M. Woodward, Chemistry: The Central Science, Pearson Prentice Hall, 2012. https://experts.illinois.edu/en/publications/chemistry -the-central-science-12th-edition (accessed August 2, 2019).

[11] Y.B. Acar, R.J. Gale, A.N. Alshawabkeh, R.E. Marks, S. Puppala, M. Bricka, R. Parker, Electrokinetic remediation: basics and technology status, Journal of Hazardous Materials. 40 (1995) 117-137.

[12] D.H. Gray, J. Schlocker, Electrochemical alteration of clay soils, Clays and Clay Minerals. 17 (1969) 309-322.

[13]D.H. Gray, Electrochemical Hardening of Clay Soils, Géotechnique. 20 (1970) 81-93.

[14] K.Y. Lo, I.I. Inculet, K.S. Ho, Electroosmotic strengthening of soft sensitive clays, Canadian 
Geotechnical Journal. 28 (1991) 62-73.

[15] S. Micic, J.Q. Shang, K.Y. Lo, Y.N. Lee, S.W. Lee, Electrokinetic strengthening of a marine sediment using intermittent current, Canadian Geotechnical Journal. 38 (2001) 287-302.

[16]C.-Y. Ou, S.-C. Chien, Y.-G. Wang, On the enhancement of electroosmotic soil improvement by the injection of saline solutions, Applied Clay Science. 44 (2009) 130-136.

[17] C.-Y. Ou, S.-C. Chien, C.-C. Yang, C.-T. Chen, Mechanism of soil cementation by electroosmotic chemical treatment, Applied Clay Science. 104 (2015) 135-142.

[18] S.-C. Chien, C.-Y. Ou, Y.-C. Lee, A novel electroosmotic chemical treatment technique for soil improvement, Applied Clay Science. 50 (2010) 481-492.

[19] C.-Y. Ou, S.-C. Chien, Y.-T. Syue, C.-T. Chen, A novel electroosmotic chemical treatment for improving the clay strength throughout the entire region, Applied Clay Science. 153 (2018) 161-171.

[20] V. Jeyakanthan, C.T. Gnanendran, S.-C. Lo, Laboratory assessment of electro-osmotic stabilization of soft clay, Canadian Geotechnical Journal. 48 (2011) 1788-1802.

[21]D.H. Gray, Electrochemical Hardening of Clay Soils, Géotechnique. 20 (1970) 81-93.

[22]L. Casagrande, Stabilisation of soils by means of electro-osmosis, Journal of the Boston Society of Civil Engineers, ASCE. 69 (1983) 255-302. 